\title{
Reform of embedded course oriented to Internet
}

\author{
He Chuan \\ School of Electronics and Information Engineering, Jingchu University of Technology, \\ Jingmen 448000,China
}

Keywords: internet; embedded system; teaching methods; reform

\begin{abstract}
In the application of the Internet technology, the embedded system plays an important role of infrastructure. Based on the analysis of the great influence of the Internet on the embedded system teaching, the teaching ideas and teaching methods of the embedded system course are adjusted according to the characteristics of the embedded system. From the aspects of distributed control, energy saving design strategy and software engineering thinking, the related knowledge points of Internet are extended. At the same time, the teaching methods are reformed to meet the needs of embedded system talents in the field of networking.
\end{abstract}

\section{Introduction}

The Internet technology and related industries have been listed as one of the strategic emerging industries in China's "the 12th Five-Year plan". It will become a revolutionary technology to improve people's quality of life and change people's life style and work style.In recent two years, nearly 100 colleges and universities in China have opened the Internet engineering major or set up the subject orientation of Internet, so as to meet the needs of professionals in the Internet. Embedded system is an important specialized course in the major of computer science and technology and automation. With the development and deepening of the Internet technology, embedded systems play an important role in the Internet, it has become an indispensable front-end platform for the realization of perception and control in the Internet applications. Therefore, the industry and academia have put forward new requirements and expectations for the teaching of embedded systems in Colleges and Universities. The author studies the main teaching forms of the embedded system course, and explores the new teaching contents and teaching modes which are beneficial to the personnel training of the Internet.

The only source of the Internet is embedded systems. Embedded system is an intelligent electronic system with embedded processor and embedded application. A comprehensive overview of the state of the matter, the normalized interface, and the infinite combination of applications are the history of the embedded system. The embedded system has a normalized architecture and a global interface, which can be embedded into the object system and coupled with physical objects to form an embedded application system.

\section{The influence of Internet of things on the teaching of Embedded System Course}

In the undergraduate course of embedded system, it is necessary to build the basic courses such as digital electronic technology, computer programming, computer composition principle, microcomputer interface and so on. With the development of embedded computing technology and wireless communication technology, the application scope of embedded system is expanding day by day, the complexity of software and hardware is also increasing, and the design thinking, technical route and reference index of the whole system are obviously dependent on the specific application background. At present, the teaching of embedded systems is broadly divided into two categories: one is to focus on hardware, and the other is to focus on software. The former is based on embedded processor, and the latter is embedded operating system. However, for most universities, the teaching of "embedded systems" can only be one of the classes due to the limited number of hours and the 
requirements of the specialized direction. With the development of embedded computing technology and micro electro mechanical sensor technology, the knowledge of embedded systems is updated quickly and has a high degree of interdisciplinary features. Therefore, the teaching of "embedded system" must follow the development of industry and technology, constantly update the teaching content, train the students to feel and understand the process of technological progress and get the ability of independent learning. However, the current "embedded system" teaching has not yet been achieved, the goal that adjusting the content of the course, teaching ideas and methods of teaching according to the development of applications and changes.

Embedded processor is a combination of computer technology, control technology and SCM technology, it is widely used in industry control, communications, electronics and portable devices because of its small size, rich integration function and many peripheral interfaces. The related courses of embedded system are usually opened by computer, automation, electronic information and other majors in Colleges and universities. The purpose is to train students to have the ability of developing embedded system software and hardware. In the context of Internet applications, the embedded system moves from "stand-alone" to "Internet", from "individual intelligence" to "swarm intelligence". Therefore, for embedded system designers, the original knowledge system is important, but it must also be clear about the new requirements, new challenges and new technologies brought by the application of Internet. As for the embedded system course in colleges and universities, we must follow the trend, adjust the content and mode of the course, and make the embedded system course more flexible and purposeful.

\section{Reform of the teaching method of Embedded System Course}

In the context of Internet applications, the integration of multi discipline and multi technology shows a clear trend, it can be said that the Internet technology is made up of embedded computing technology, sensor technology, wireless networking technology, mobile computing technology and advanced data processing technology. Among them, the most obvious feature of the Internet is the network of equipment and the distributed form of computing. To a greater extent, the networking of Internet is not the use of Internet, but the use of wireless network technology. The distributed characteristics of the Internet need to emphasize how to complete the computing task under the condition of limited resources. In addition, with the development of embedded computing technology and wireless communication technology, the cost of hardware continues to decline, and the cost of the system is increasingly affected by the cost of software. Whether for embedded systems of single devices or networked embedded systems,system software and application software become more and more important in the whole system's function and status. However, the embedded system course can not undertake all the teaching tasks about the system platform in the whole Internet. Therefore, in view of the several important core features of the application design and development of the Internet, the author studies from 3 aspects, and explores the teaching content and teaching methods.

1. Distributed embedded control. For the teaching of distributed embedded systems, communication protocols and their behaviors need to be understood first. Only in this way can we understand how to ensure real-time performance on the whole system level. Under the guidance of this approach, important topics such as message broadcasting and time synchronization should be added to the "embedded system" course. These are the keys to correctly analyzing the performance of embedded systems.

The difficulty of this part is mainly embodied in the students' general knowledge of the distributed system,besides, it is not easy to come into contact with a large scale test platform to form the necessary perceptual knowledge in the form of distributed embedded systems and their performance evaluation methods. Therefore, the simulation software can be used properly, which is helpful for teaching.

2. Energy efficiency oriented embedded system. For this part of the teaching content, first of all,we should let students make clear why the management of energy consumption should be 
considered in the design process, and why the typical energy-saving technologies should be emphasized. For example, the use of low-power processors and radio chips, the design of advanced network protocols to reduce the number of transmissions of a single device, and so on. It is also important to avoid telling technologies that rely heavily on hardware platforms or for specific applications. In addition to the specific energy-saving technologies, we should also increase the overall energy consumption reduction program at the whole system level. Only in this way can students not only master the specific energy-saving technologies, but also understand the macro energy saving design ideas.

This part of the teaching is easy to obtain the reference books, but there is a problem with the reference book: The technical system of energy-saving system is too complete and is not suitable for studying in the course of "embedded system". Therefore, it is more effective to select academic papers corresponding to specific energy-saving technologies as supplementary materials after class.

3. The thinking of software engineering. Although the embedded system design is very dependent on the application and the hardware structure, however, with the development of embedded computing technology, the development of embedded system has gradually become an application development based on embedded operating system.

During teaching the content of software engineering, the most important thing is to train students the core idea of the life cycle of "requirements analysis, design, implementation, verification, implementation , maintenance". The teacher can analyze the typical development case in the industry and analyze the influence of the core part of the system development on the whole system design. It should be noted that this part of teaching is to train the students' basic thinking of software engineering.

\section{Construction of embedded system course}

At present, there are many teaching materials for embedded systems and Linux programming at home and abroad, so it is very important to choose appropriate teaching materials. It is worth mentioning that many foreign classical textbooks have corresponding Chinese versions, but their translation levels are different. Because of the complexity of the embedded system, teachers should choose appropriate cases to explain. Teachers should encourage students to study independently, and make them know the modern teaching thought of "teacher led and student oriented".

After careful analysis, the author suggest that students using the English original edition of $<$ Programming Embedded System $>$ written by M.Barr and A.Massa as an introductory reading of this course.At the same time,students can use the book $<$ Building Embedded Linux System $>$ written by K.Yaghmour as a reference material for learning embedded Linux programming and building embedded systems.

\section{Conclusion}

Discussion on new teaching contents and teaching methods of "embedded system" course in the context of Internet are to improve the students' ability to analyze, design and implement in the field of embedded systems. For different kinds of knowledge and problems, students in different classes show different levels of attention and different solutions. As a result, teachers can make up a small group of 3 5 students with similar interests,they can put good students and less capable students into a team so that the students can start their own collaborative learning,discuss and make progress together. In addition, there is still a sense of competition between teams in the face of the same design topic, which can promote the whole class learning atmosphere, and help to improve the practical ability of all students.

This course is based on the knowledge system of embedded system and is based on the needs of personnel training in the Internet. According to the characteristics of the Internet curriculum system, taking the hardware and software structure of embedded system as the main line, starting with the distributed characteristics of network embedded system and the demand for energy efficiency of the system,we pay attention to training students to use appropriate software engineering thinking to 
analyze and solve the problems faced in the development of embedded systems.

It is impractical to teach the embedded system knowledge related to the Internet in class, the author hopes to make up for the deficiencies of the "embedded system" course by discussing the teaching contents and teaching methods of the embedded system. Enable students to have the right thinking direction and basic development skills in the design of Internet embedded systems in the future.

\section{References}

[1]Huang Yuyue,Chang Jinyi,Xu Xiulan. Research on practical talents training mode of Internet[J]. Computer education,2012(23):58-62.

[2]Wang Sufeng,Ning Hong,Lu Hongyi. The exploration of embedded system curriculum system and its innovative practice[J]. Computer education,2010(7):45-47.

[3]Tian Ze. Development and application of embedded system[M].Beijing: Beihang University press,2005:5-15.

[4]Huo Hua.Rerorm of embedded technology course[J]. Computer education,2012(6):48-50.

[5]KOOPMAN P,CHOSET H,GANDHI R,et al.Undergraduate embedded system education at Carnegie Mellon[J].ACM Trans.on Embedded Computing Systems,2005,4(3):500-528. 